EDGE EFFECTS ON FORCES AND MAGNETIC FIEIDS PRODUCED BY A CONDUCTOR MOVING PAST A MAGNET

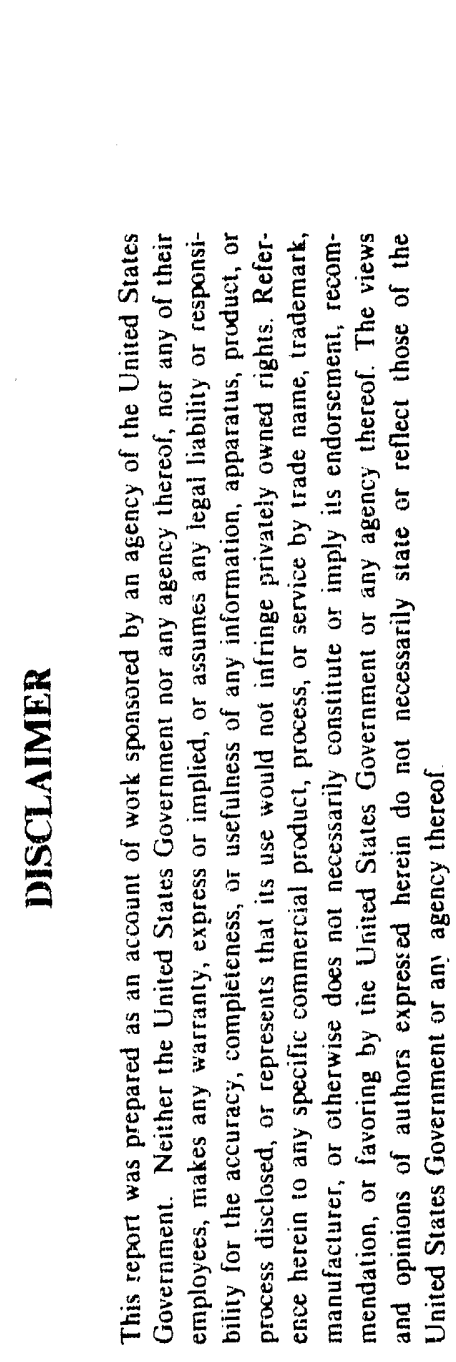

\author{
T. M. Mulcahy, J. R. Hull, and J. D. Almer \\ Argonne National Laboratory, Argonne, IL 60439
}

ANL/CP- -74708

DE92 010502

T. D. Rossing

Northern Mlinois University, DeKalb, IL 60115

The slubrnitted manuscripl has buen authored
by a contractur of the U. S. Government under
contract No. W-31-109-ENG-38. Accordingly.
the U. S. Government retains a nonexclusivg.
royalty-tree Ikense to publish or moroduce the
pubilshed form of this contributlon, or allow
others to do so, lor U. S. Government
purpeses.

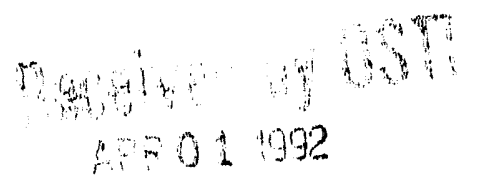

To be presented at International Magnetics Conference, Intermag '92, St. Louis, April 13-16, 1992, and published in IEEE Transactions on Magnetics

Work at Argonne National Laboratory was sponsored by the U.S. Army Corps of Engineers in accordance with the Military Interdepartmental Purchase Request \# E8691R001 to the U.S. Department of Energy, Office of Transportation Technologies.

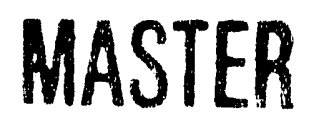




\title{
EDGE EFFECTS ON FORCES AND MAGNETIC FIELDS PRODUCED BY A CONDUCTOR MOVING PAST A MAGNET
}

\author{
T. M. Mulcahy, J. R. Hull, and J. D. Almer \\ Argonne National Laboratory, Argonne, IL 60439 \\ T. D. Rossing \\ Norhem Illinois University, De Kalb, IL 60115
}

\begin{abstract}
Experfments bave been performed to further understand the forces actlog on magnets moving along and over the edge of a continuous conducting sheet and to produce a comprehensive data set for the valliation of analysis methods. Mapplng the magnetic fleld gives Information about the eddy currents induced in the conductor, which agrees with numerical calcualtions.
\end{abstract}

\section{INTRODUCTION}

Typically, rectangular-shaped, superconducting magnets are placed on moving vehicles to induce eddy-currents in guideway conductors and achieve levitation by repulsion (Lenz's law). The associated levitation or lift forces $F_{L}$ created by the opposing magnetic field of the eddy currents, and drag forces $F_{D}$, created by eddy current losses, are well understood for infinite conducting sheets [1]. Conductors can be mounted on the side walls of U-shaped guideways to provide lateral guidance forces $F_{G}$ that are also predictable, if the assumption of infinite conducting sheets is valid.

Altematively, guidance forces can be created in flat guideway designs, if the vehicle magnets are located near the edge of the conducting sheets [2-6], as shown in Fig. 1. In one of the earliest experimental and theoretical investigations of edge effects [3], $F_{L}, F_{D}$, and $F_{G}$ were determined for a rectangular superconducting magnet held stationary over a rotating aluminum drum at a constant coil height $h$ and several $Y^{*}>0$ The drum was solid and simulated a very thick $(T=\infty)$ and fast moving $(140 \mathrm{~m} / \mathrm{s})$ guideway of width $W . F_{L}$ was found to be reduced only slightly $(20 \%)$ as the coil was moved from the centered to the edge-aligned position $\left(Y^{*}=0\right)$, while $F_{G}$ increased from 0 to $30 \%$ of $F_{L}$. Also, $\left(F_{L}+F_{G}\right) / F_{D}$ remained constant for all lateral posicions, and edge effects were significant only when $Y^{*}<h$ to $2 h$.

In some dual-strip guideway designs [4,5], superconducting magnets provide lift, guidance, and propulsion. Typically, the magnets are centered over and span the gap between the strips, with only the fringe fields at the edge of the magnets interacting with the strips to provide lift and guidance forces $\left(\gamma^{*} \leq-1.9 a\right)$. For such small overlap of the magnet and guideway $(\leq 5 \%)$, the measured $F_{L}$ were only $3 \%$ of the lift that would be provided by a wide conducting sheet where edge effects can be considered negligible. However, for only a small lateral displacement of the magnet from its' centered position, $F_{G}$ as large as the $F_{L}$ were developed. In comparison to the results for a wide conducting sheet, no drag

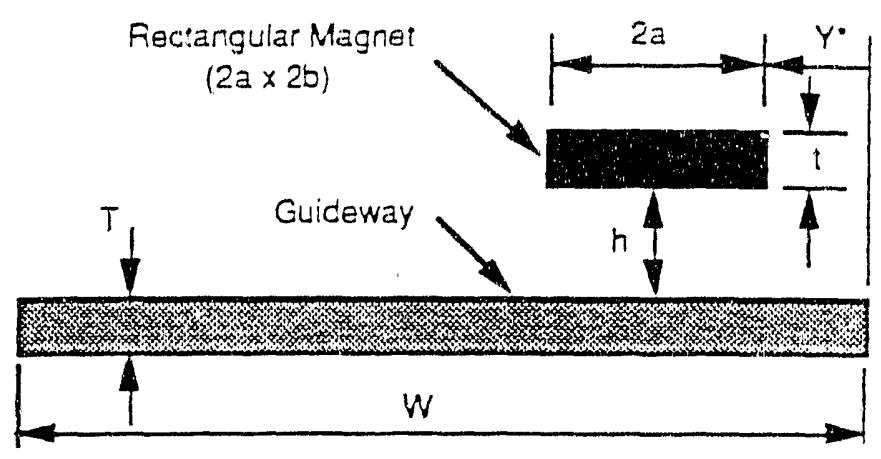

Fig. 1. Magnet offset toward one edge of the guideway.

peaks were observed and the $F_{U} / F_{D}$ were reduced and sensitive functions of the overlap of the edge of the magnet and the strip, $\left(Y^{*}+2 a\right)$.

In contrast, in an analysis [6] of a closely spaced linear array of alternating polarity magnets over dual-strips, slight drag peaks were found and $F_{V} F_{D}$ became larger as the overlap of the magnets and the strips were minimized. In another extensively studied design [2], magnets were centered over each of the strips to produce lift, and nuil-flux coils were located under the separate motor magnets spanning the gap to enhance the guidance forces. Apparently, the design possibilities are many and the generation of the forces can be complex. A verified analysis tool seems a necessity.

The purpose of this experimental study is to further investigate edge effects and provide well defined experimencal results that can be used for validation of analysis methods. This study is patterned after the work in [3], except the geomerty parameters are extended to cover the range of the dual-surip guideway designs $\left(Y^{*}<0\right)$.

\section{EXPERIMENTAL APPARATUS}

We measured ine steady-state forces acting on a rectungular FeBNd permanent magnet positioned above a 6061 -T6 aluminum rim atsoched to a 1.2 -m-diameter flywheel moving at surface velocities up $1036 \mathrm{~m} / \mathrm{s}$. The test parameters werc: $2 \mathrm{a}=25.4 \mathrm{~mm}, 2 \mathrm{~b}=50.8 \mathrm{~mm}, \mathrm{~T}=\mathrm{t}=6.4 \mathrm{~mm}, \mathrm{~W}=101.6$ $\mathrm{mm}, \mathrm{h}=5$ to $12.7 \mathrm{~mm}$, and $\mathrm{Y}^{*}=38.1$ to $-38.1 \mathrm{~mm}$. Out of roundness of the rim was measured to be less than $\pm 0.8 \mathrm{~mm}$. The magnet was held stationary by a two-component force transducer, comprised of two BLH C2Gl load cells connected in series. To measure all three force components, two 


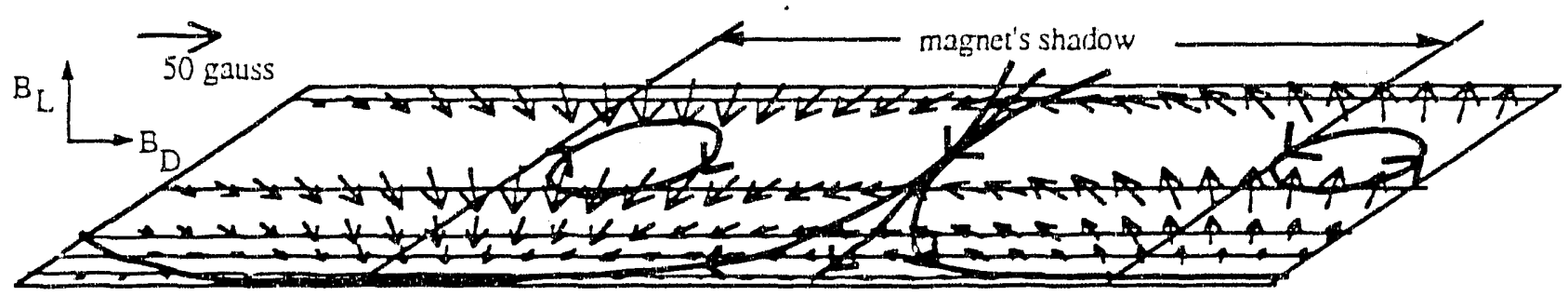

(a)

Guideway velocity -.......->

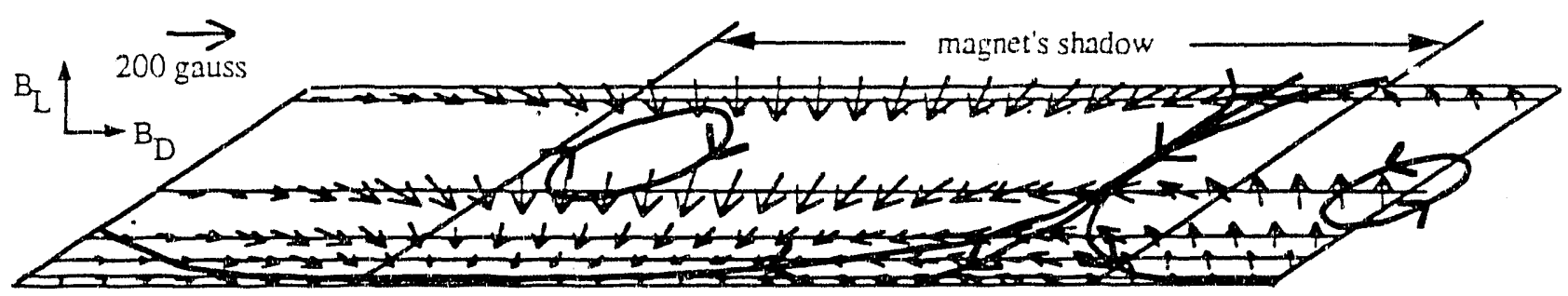

(b)

Fig. 3. The magnetic field in the lift and drag directions at $5.9 \mathrm{~mm}$ above the guideway for $Y^{*}=0$, (a) $V=4.5 \mathrm{~m} / \mathrm{s}$, an . (b) $\mathrm{V}=36.1 \mathrm{~m} / \mathrm{s}$.

as was found for $Y^{*} \geq 0[3]$. The sensitivity of the forces to the surface area of the edge of the conductor remains to be determined, especially at high velocities. For increasing velocities, the shifts toward the rear of the two opposing guideway eddy-current loops were inhibited over all or part of the current field, depending on the amount of overlap of the magnet and the guideway.

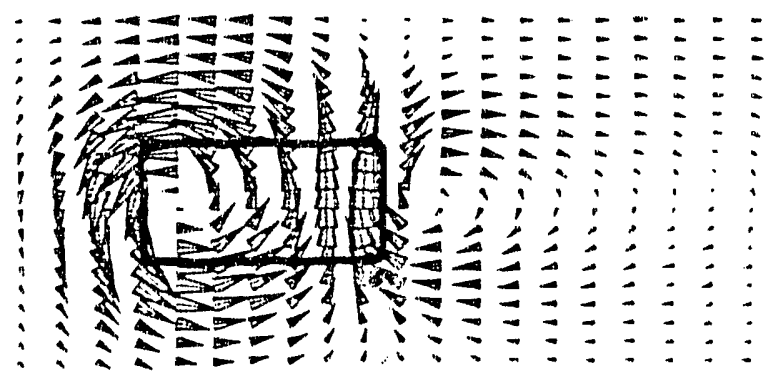

(a)

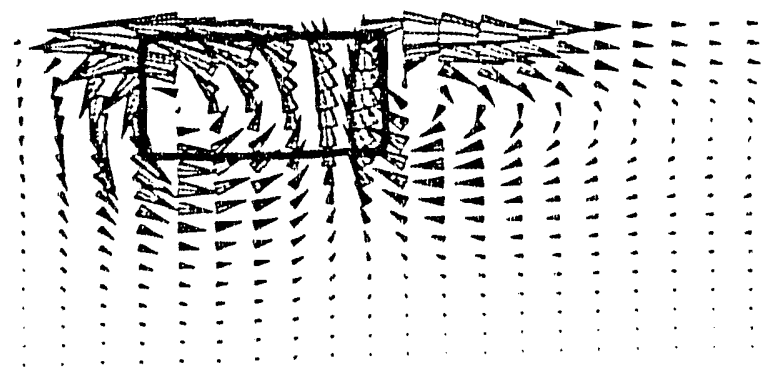

(b)

Fig. 4 Eddy currents distribution just below top surface of the guideway for $V=40 \mathrm{~m} / \mathrm{s}$ (a) $Y^{*}=25.4 \mathrm{~mm}$ (b) $Y^{*}=0$. The Coil is moving left.

\section{ACKNOWLELGEMENT}

The authors are indebted to Frank Moon of Cornel! University for the flywheel used in testing and to $D$. Yacobellis and L. Chavez for assistance with the measurements. Work at Argonne National Laboratory was sponsored by the U.S. Army Corps of Engineers in accordance with the Military Interdepartmental Purchase Request \# E8691R001 to the U.S. Deparment of Energy, Conservation and Renewable Energy, Cffice of Transportation Technologies.

\section{REFERENCES}

[1] I. Reizz L. C. Davis. "Forre on a rectungular cuil moving above a conducting slab," J. Appl. Phys., vol. 43, pp. 1547-1553, 1972.

[2] D. L A Aherion and A. R. Eastharn. Superconducting maglev and LS.S development in Canada, IEEE Trans. Magn., yol. 11, pp. 627, 1975

[3] R. H. Burchers and L C. Davis, "Force on a coil moving over a conducting surface including edge and channel effects," $J$ of $\mathrm{dFF}$. Phys., vol. 43, pp. 2418-2427, 1972.

[4] J. L Mahtani and R. G. Rhodes, "Electrodynamic force characterization of the split-track maglev system, "Journat de Physique, Colloque C1 45, pp. C1 747-C1 751, 1984.

[5] J. L. Mahcani and R. G. Rhodes, "Experimental studies of the superconducting split-lsack maglev system," Internationd Conference on Moglev Transport 85, pp. 199-205. 1985.

[6] T. Sakamoto, A. R. Eastham, and G.E. Dawson, "Induced currents and fores for the split-guideway eletrodynamic levitation system," IEEE Trans. Magn., in press.

[7] J. L. Mahatani and J. L. Rhodes, "Eddy current distributions for the Warwick splir-track maglev configuration," IEEE Trans. Magn., vol. 18, pp. $1740-17+2,1982$.

[8] Vector Fields, Lid., 24 Bankside, Kidlington, Oxford OX5 IJE, England 
separate tests, with different orientations of the force transducer, had to be performed for each position of the magnet and velocity. Duplication of force measurements provided a measure of the experimental accuracy.

Magnetic fields for the centered, edge-aligned, and 25\%. overlap positions of the magnet, $Y^{*}=38.1,0$, and -28.6 mm; respectively, were measured by moving a singlecomponent F.W. Bell (HTG-1-0608) gaussmeter probe parallel to the centerlines and longitudinal edges of the magnet. The sensing area of the Hall-effect element was less than $2-\mathrm{mm}$ in diameter. All three field components (in the lifh drag, and guidance directions) were measured for a magnet height of $12.7 \mathrm{~mm}$ and a probe height of $5.9 \mathrm{~mm}$. The magnetic fields due to the eddy currents induced in the moving guiderway were determined by suburacting the magnetic fields with the flywheel at rest from the fields with the flywheel moving.

Our inability to exactly position the magnet and gaussmeter probe, with respect to the guideway and each other, dominated our experimental error, estimated to be $\pm 5 \%$.

\section{DISCUSSION OF RESULTS}

Force measurements were made for the centered magnet position $\left(\mathrm{Y}^{*}=38.1 \mathrm{~mm}, \mathrm{FG}_{\mathrm{G}}=0\right)$ and compared to available closed form solutions for a single current-carrying coil [1] at different coil currents. A 25.4- x 50.8-mm current element carrying $6000 \mathrm{~A}$, and located at the center height of the magnet, gave excellent quantitative fits to the force measurement data for all heights of the magnet and velocicis.

The measured lift and drag forces were found to decrease by one to two orders of magnitude as $Y * / h$ was decreased and the magnet was moved off the edge of the guideway. Figure 2 shows typical results at $\mathrm{V}=36.1 \mathrm{~m} / \mathrm{s}$ and $\mathrm{h}=12.7 \mathrm{~mm}$. The maximum guidance force occurred when the edge of the magnet and guideway were nearly flush. In contrast to the experimental results for a thick guideway and the theory for a thin guideway in the high-speed limit [3], the lift force in Fig. 2 is attenuated wo to three times more by the presence of the edge at low speeds. This suggests the eddy-current skin depth and the surface area of the edge are both important

The force ratios $\left(F_{L} / F_{G}\right)$ and $\left[\left(F_{L}+F_{G}\right) / F_{D}\right]$ decreased significantly as the inagnet was moved off the edge of the guideway. The FL/FD ratio recovered slightly when the magnet was almost completely off the guideway $\left(Y^{*}<-2 h\right)$. The $\left(F_{L}+F_{G}\right) / F_{D}$ ratio was nearly constant when the magnet and guideway completely overlaped $\left(Y^{*} \geq 0\right)$, as previously shown [3], and in the region of $F_{L} / F_{D}$ recovery, as previously suspected [5]. The recovery region was identified [5] as optimal for the design of dual-strip guideways using gap-spanning magnets only, although the data suggest that positions with more magnet and guideway orerlap can produce larger guidance forces and lift-to-drag ratios.

Magnetic field measurements for $Y^{*}=0$, shown in Fig. 3. suggest that the eddy currents are concentrated by and at the edge of the guideway. Eddy-current directional patterns in the guideway were extrapolated from the magnetic field data and added to Fig. 3. Clearly, some of the eddy currents must

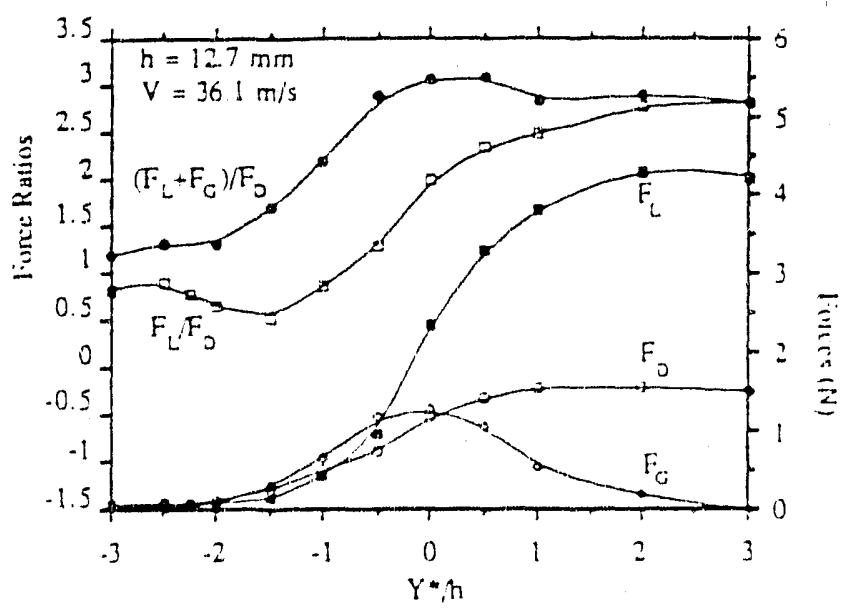

Fig. 2. The effects of the procimity of the guidesuay edge on the magnetic forces and their ratios

flow on the narrow edge of the conductor, especially at higher velocicies.

For a velocity increase from 4.5 to $36.1 \mathrm{~m} / \mathrm{s}$, the shift toward the rear of the eddy currents near the edge (the transition to the high-speed limit) was somewhat inhibited, as previously observed [7] for all the eddy currents at a very small overlap of the magnet and guideway, $\mathrm{Y}^{*}=-1.9 \mathrm{a}$. The eddy curtents away from the edge of the rim showed more of a shift. Smaller shifs wre observed for $Y^{*}=-28.1 \mathrm{~mm}$, and, again, the eddy currents closer to the edge were inhibited more. Finally, the disappearance of distinct drag force peaks ware observed and found whenever the guidance forces and inhibition of the eddy-current shift were significant.

The above results are corroborated by finite-element calcylations of the same system using the computer code ELECTRA [8], where we modeled the magnet as a rectangular coil with the same outside dimensions. $F_{L}, F_{D}$, and $F_{C}$ calculated by ELECTRA agreed with the measured $f$ rees to within $10 \%$. Eddy currents in a plane just below the upper surface of the guideway are shown in Fig. 4. When the coil is at the edge $\left(Y^{*}=0\right)$ the eddy current vortex is much closer to the comer of the coil than when the coil is near the center of the guideway $\left(Y^{*}=25.4 \mathrm{~mm}\right)$. This behavior is also seen for $Y^{*}<0$ and could account for the absence of a drag peak as the velocity increases. Physically, we attribute the fixation of the vortex center as due to rapid diffusion of the highdensity current along the edge in the wake of the coil.

For $Y^{*}<0$, examination of the current density show's a tendency for the current to run along the guideway edge. This suggesis $F_{G}$ could be increased by thickening the guideway along tha edge.

\section{CONCLUSIONS}

An FeBNd permanent magnet was successfully characterized and used to study forces the developed on it at the edge of a moving conductor sheet. The force ratio $\left(F_{L}+F_{G}\right) / F_{D}$ cannot to be assumed constant for $Y^{*} \leq 0$. 

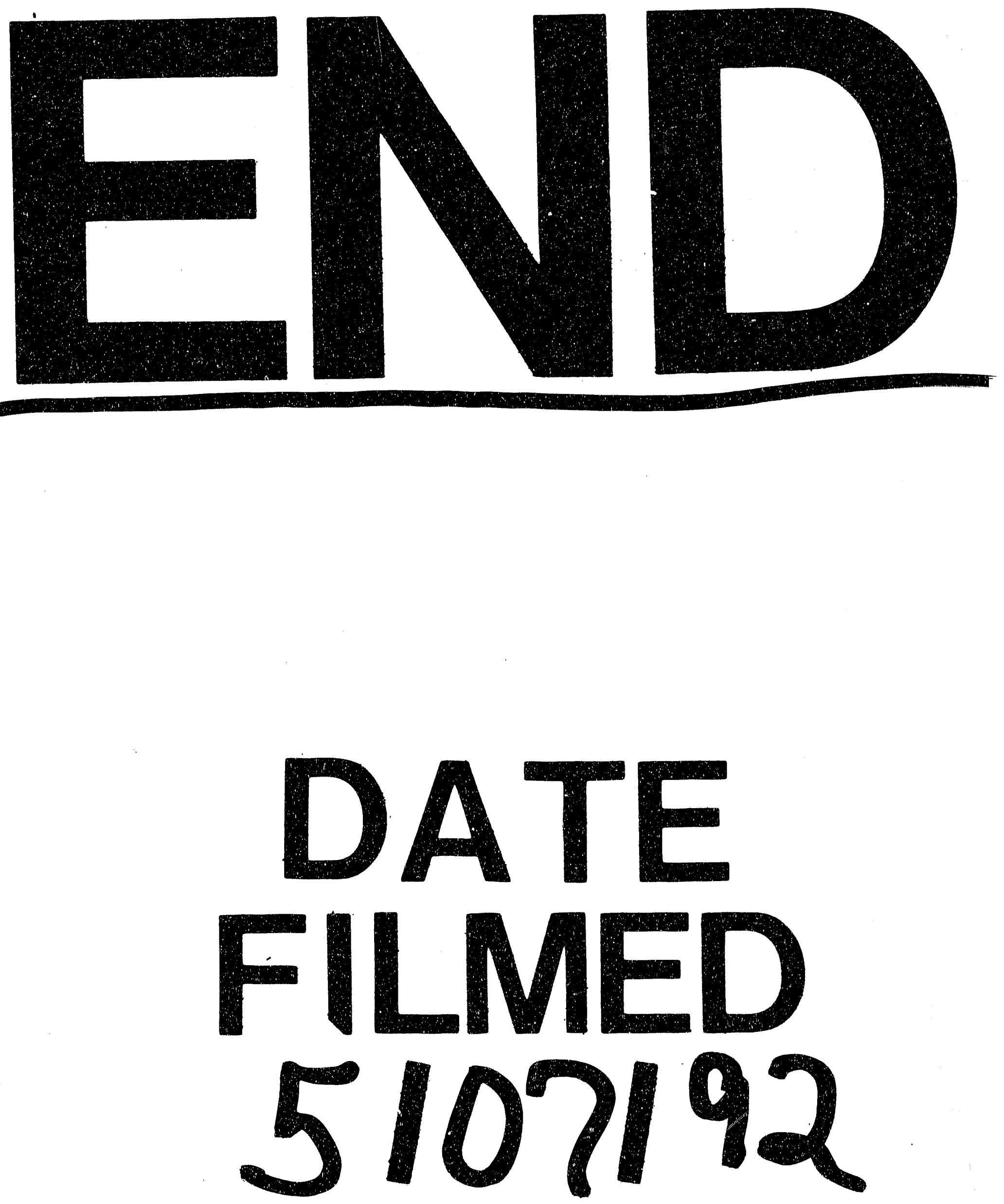
